ketamine. Piloting of the proposed ketamine sedation policy confirmed feasibility of its use within the Paediatric Emergency Department. The process from administration of ketamine to end of casting took $11 \mathrm{~min}$. The patient was able to recover in the short stay ward and after review, was discharged home. Conclusion Large numbers of patients, who are currently returning for manipulation under general anaesthesia, would benefit from the implementation of ketamine sedation. This would help to reduce burden on the orthopaedic ward, theatre space, and anaesthetic team. This method of sedation also carries less risks than general anaesthesia. It is anticipated that if changes are made to the emergency department layout and orthopaedic team staffing it will be feasible to carry out ketamine sedation 3 times a week. The next stage would be to carry out a week-long pilot to accurately assess the process before full implementation.

\section{G288(P) EVALUATION OF PATIENT ADMISSIONS THROUGH A PAEDIATRIC EMERGENCY DEPARTMENT AT A CENTRAL HOSPITAL IN A RESOURCE-POOR SETTING}

J Rasquinha, J Langton. Department of Paediatrics, Queen Elizabeth Central Hospital, Blantyre, Malawi

\subsection{6/archdischild-2018-rcpch.280}

Aims To evaluate patient attendance, admission rates and use of the resuscitation room in a Paediatric Emergency Department at a central hospital in a resource-poor country. To identify how trends have changed and if there is a need to change the clinical environment to match the requirements of patients seen.

Methods Data was collated from departmental daily summary books, computerised and analysed to evaluate attendance, admission and use of the resuscitation room. A 1 month period was analysed in more depth using additional resuscitation room and admission record books to review patient demographics and diagnoses.

Results Records were collected from September 2014 - May 2017 and grouped to identify year-on-year trends. Some dates had missing information so averages have been used to account for this.

There are approximately 40000 total attendances and 11000 admissions per year through the department. Whilst the number of admissions overall appears to be falling, the rates of those admitted through the resuscitation room appear to be increasing, from an average of $17 \%$ in $2014 / 15 \%$ to $22 \%$ in $2016 / 17$. In $2016 / 17$ the monthly percentage of admissions through the resuscitation room ranged from 17\%-30\%.

In evaluating a 1 month (January 2017) snapshot of 1007 admitted patients, over a quarter were under the age of 12 months. $24.9 \%$ patients admitted during this month were seen in the resuscitation room and the most common diagnoses of these patients were Malaria and/or Anaemia, Bronchiolitis and Pneumonia.

Conclusion Admissions through the department appear to be decreasing, but the proportion of sick children being assessed in the resuscitation room is increasing. This may reflect that patients with milder illnesses are now being managed better in the district hospitals, with only those who are exceedingly sick or requiring specialist services being referred.

This review identified that approximately $22 \%$ of paediatric patients admitted are currently coming through the three- bedded resuscitation room, and due to the severity of their symptoms and the amount of clinical care required, this certainly identifies a justification for redesign and expansion of this resuscitation room.

\section{G289(P) REFLECTIONS ON A SHORT-TERM PAEDIATRIC MEDICAL MISSION TO A SYRIAN REFUGEE CAMP IN JORDAN}

M Mazlan, S Bihari. Paediatrics and Child Health, Wales Deanery, Cardiff, UK

\subsection{6/archdischild-2018-rcpch.281}

Aims The Syrian refugee crisis is entering its 7 th year. There are various humanitarian organisation are on the ground conducting short-term medical missions. We aim to describe the clinical care that was delivered and my personal reflection on a short-term medical mission to Jordan with the Syrian American Medical Society (SAMS).

Methods Through the 6 day mission, we held 6 paediatric clinics each in various locations across Jordan including the Al-Zataari refugee camp near the Syrian border. We gathered prospective quantitative data including age, presenting complaint, diagnosis, management and prescription. The qualitative data is my own personal reflection on the mission.

Results We saw a total of 384 patients with an average of 32 patients per day. The average consultation time is $10 \mathrm{~min}$ per patient. The most common presenting complaint is sore throat with or without fever (41\%). 30\% of presenting complaint is related to poor growth or lack of appetite. The most common primary diagnosis is viral upper respiratory tract infection (60\%). Significant diagnosis include secondary nocturnal enuresis (10 patients), rickets (1 patient), severe pneumonia and dehydration (1 patient), untreated cleft lip and palate (1 patient). Almost $75 \%$ received prescription for paracetamol. Amoxicillin was dispensed to $21 \%$ of patients. The unwell patient with pneumonia and dehydration was stabilised locally then sent to the referral hospital. The outcome of her progress is unknown. The patient with untreated cleft was referred via SAMS to a charity specialising in cleft repair. She received her operation a week after the mission.

Conclusion The high rate of well children with acute respiratory disease is attributable to living conditions and desert environment. Rigid dispensing of amoxicillin is important to prevent unnecessary use of antibiotics especially as they are able to purchase without prescription. Trauma is the most likely cause of nocturnal enuresis and the need for mental health support cannot be underestimated. Assessment of anaemia and vitamin D deficiency is prime to avoid chronic complications. While the mission is rewarding personally, there can be further improvements to create a more sustainable model of care. Trainee participation in such missions should be encouraged to raise awareness of refugees' plight and for clinical experience.

\section{G290(P) LINKING PAEDIATRIC ONCOLOGY RESEARCH WITH ELECTIVES}

${ }^{1} \mathrm{RC}$ Mansfield, ${ }^{2} \mathrm{~K}$ Ford, ${ }^{2} \mathrm{~K}$ Lakhoo, ${ }^{1} \mathrm{~L}$ Gardner, ${ }^{1} \mathrm{G}$ Ndukwe, ${ }^{3} \mathrm{H}$ de Berker. ${ }^{1} \mathrm{Oxford}$ Medical School, University of Oxford, Oxford, UK; ${ }^{2}$ Paediatric Surgery, Oxford University and Oxford University Hospitals, Oxford, UK; ${ }^{3}$ Cardiff and Vale University Health Board, University Hospital of Wales, Cardiff, UK

10.1136/archdischild-2018-rcpch.282 
Aims Since the publication of the Lancet commission in global surgery (2015) the need for more timely, accessible and affordable surgical care in low- and middle-income countries (LMIC) has been increasingly recognised. There is a paucity of data to guide healthcare advocacy and resource allocation. This student paediatrics society has collaborated with UK paediatric surgeons to develop a novel research hub (OxPLORE). OxPLORE will investigate three paediatric solid tumours (Wilm's tumours, Neuroblastomas and Rhabdomyosarcomas) and report their incidence, management and post-operative outcomes in differently resourced settings visited by medical students on elective placements.

Methods Students are currently forming links with consultants in their host centres, prior to the first batch of elective placements in November 2017 - February 2018. Host consultants are pairing elective students with local trainees and medical students.

Data will be anonymously recorded on a standardised proforma, by elective students and their host collaborators. The primary outcome is survival (at post-operative day 28, date of death, or date last seen alive). Secondary outcomes include: patient demographics; tumour stage; pre-operative management and morbidity (Clavien-Dindo Classification); operative details; post-operative management and follow up.

Ethical approval is arranged for each centre at an institutional level. All collaborators will be named authors on resulting publications.

Results In November and December, the project supervisors will run the first data collection pilot in Tanzania, and a student participant will gather data in Rwanda. Further OxPLORE collaborations will follow in early 2018.

The data from our UK centre has already been gathered (from 2012-2017). These data will be analysed and compared to Tanzanian and Rwandan data to describe the differing experiences of paediatric solid tumours in these centres.

Conclusions

- Information generated from these collaborations will inform individual centres of which tumours represent a greater burden of disease, and act as an advocacy tool to be used to obtain funding for surgical resources.

- OxPLORE students will develop relationships with link institutions, which will be nurtured for future collaborations driven by local colleagues.

- Comparative data may help to advise best management of paediatric solid tumours in differently resourced settings globally.

\section{G291(P) UNINTENTIONAL CHILDHOOD INJURIES IN SUB SAHARAN AFRICA - A FOCUSSED LITERATURE REVIEW 2009-2015}

B Eder, D Magnus. The School of Social and Community Medicine, University of Bristol, Bristol, UK

\subsection{6/archdischild-2018-rcpch.283}

Aims Childhood injury disproportionately affects children in low and middle-income countries and Sub Saharan Africa has the highest death rate in the world from injury. Given the burden of childhood injury in Sub Saharan Africa the allocation of resources for public health interventions to address this problem are inadequate. This focussed literature review aimed to describe the burden of childhood injury in Sub
Saharan Africa and to explore the extent to which trauma registries or injury databases are being used by focussing on research conducted following the Report on Child Injury Prevention.

Methods A search protocol was designed using the key terms 'child* or paediatric or paediatric' AND 'injur* or trauma' AND 'unintentional or accident".' The literature was reviewed for all studies in Sub Saharan Africa as defined by the United Nations. The databases Medline, Pubmed and Web of Science were searched for papers in the English Language that have been published since 2009. Twenty eight papers were identified for full text review and thirteen papers met the inclusion criteria. Data were extracted from the papers and common themes discussed.

Results Six countries out of the fifty one in Sub Saharan Africa had published papers on childhood unintentional injury since 2009. A large degree of heterogeneity existed between the studies. There were significant differences in the proportion of unintentional injuries due to either falls or road traffic incidents depending on the location of the study. Burns were found to be most common in children under the age of 5 whilst boys in all studies were more at risk of injury. Children were more likely to be injured at home or on the streets as opposed to school.

Conclusion Childhood unintentional injury in Sub Saharan Africa is under researched. Greater efforts need to be made to carry out research at a local level in order for public health interventions to produce the most benefit. The widespread implementation of trauma registries will help this process.

\section{G292(P) ESTIMATES OF THE BURDEN OF GROUP B STREPTOCOCCAL DISEASE WORLDWIDE FOR PREGNANT WOMEN, STILLBIRTHS AND CHILDREN}

${ }^{1} \mathrm{AC}$ Seale, ${ }^{1} \mathrm{~F}$ Bianchi-Jassir, ${ }^{1} \mathrm{NJ}$ Russell, ${ }^{1} \mathrm{M}$ Kohli-Lynch, ${ }^{1,2} \mathrm{CJ}$ Tann, ${ }^{3} \mathrm{~J}$ Hall, ${ }^{4} \mathrm{~L}$ Madrid, ${ }^{1}$ JE Lawn. ${ }^{1}$ MARCH Centre, London School of Hygiene and Tropical Medicine, London, UK; ${ }^{2}$ Neonatal Medicine, University College London Hospital, London, UK; ${ }^{3}$ Institute for Women's Health, University College London, London, UK; ${ }^{4}$ IS Global, University of Barcelona, Barcelona, Spain; ${ }^{5}$ Bayor College of Medicine, Houston, Texas, USA

\subsection{6/archdischild-2018-rcpch.284}

Background We aimed to estimate, for the first time, the global burden of Group B Streptococcus (GBS), with regards to invasive disease in infants, as well as in pregnant and postpartum women, and fetal infection/stillbirth. Intrapartum antibiotic prophylaxis (IAP) is currently used for prevention of early onset infant disease in high-income contexts, but is difficult to implement globally, and may contribute to antimicrobial resistance. Maternal GBS vaccines are in development.

Methods For 2015 live births, we used data from systematic reviews and meta-analyses (presented in separate papers in this GBS supplement) and a compartmental model to estimate:

- exposure to maternal GBS colonisation,

- cases of infant invasive GBS disease,

- deaths, and

- disabilities.

We applied incidence or prevalence data to estimate cases of maternal and fetal infection/stillbirth, and infants with invasive GBS disease presenting with neonatal encephalopathy (NE). We applied risk ratios to estimate numbers of preterm births attributable to GBS. Uncertainty was also estimated. 